\title{
Die Belegerteilungspflicht als Verursacher von Umwelt- und Gesundheitsgefährdungen?
}

\author{
Marco Genslein · Anke Bockreis
}

Online publiziert: 21. Oktober 2016

(c) Der/die Autor(en) 2016. Dieser Artikel ist eine Open-Access-Publikation.

\section{Zusammenfassung Die am 01.01.2016 in Kraft getretene Belegerteilungspflicht erfordert in Österreich die Ausstellung eines Kassenbelegs für jede Barzah- lung. Als Kassenbelege finden haupt- sächlich Thermopapiere Anwendung, die Farbentwickler wie beispielswei- \\ mopapiere als Hauptverursacher von Belastungen durch BPA. \\ Mandatory receipts as a source of environmental and health hazards?} se Bisphenol A (BPA) enthalten. Von diesen gehen erhebliche Gesundheitsund Umweltgefahren aus. Die vorliegende Arbeit soll klären, inwieweit die Belegerteilungspflicht die Risiken für Gesundheit und Umwelt erhöht. Studienergebnisse belegen, dass BPA als Verursacher für Herz-Kreislauf-Erkrankungen oder für die Beeinflussung der Entwicklung von Mikroorganismen in Frage kommt. Untersuchungen von Kassenbelegen weisen BPA-Konzentrationen von bis $\mathrm{zu} 30.000 \mathrm{mg} / \mathrm{kg}$ in Kassenbelegen auf. Eine Abschätzung zeigt, dass die Belegerteilungspflicht den Verbrauch an Thermopapieren erhöhen kann. Österreichweit ist jedoch von einer moderaten Steigerung des Verbrauchs von Thermopapieren für Kassenbelege auszugehen. Dennoch wird eine noch größere Personenanzahl durch den permanenten Umgang mit Kassenbelegen betroffen sein. $\mathrm{Zu}-$ dem verursachen Kassenbelege, die ins Papierrecycling gelangen, Kontaminationen der neuen Papierprodukte mit Farbentwicklern. Zwar befinden sich Kassenbelege nur zu einem sehr geringen Anteil im separat gesammelten Altpapier, aber bedingt durch die besonders hohen Konzentrationen an Farbentwicklern wähnen Studien Ther-

DI (FH) M. Genslein, MSc. (凹) . Univ.-Prof. Dr.-Ing. A. Bockreis ( $\square)$ Institut für Infrastruktur, Arbeitsbereich Umwelttechnik, Fachgebiet Abfallbehandlung und Ressourcenmanagement, Universität Innsbruck,

Technikerstraße 13, 6020 Innsbruck, Österreich

marco.genslein@uibk.ac.at; anke.bockreis@uibk.ac.at
Abstract Since 01.01.2016 it has been compulsory for all Austrian businesses to issue receipts for all cash payments. Most receipts are printed on thermal paper using color developers such as bisphenol A (BPA), which pose considerable health and environmental hazards. This study investigates the extent to which the obligation to provide receipts increases the risks to health and the environment. Research has shown that BPA is a possible cause of cardiovascular diseases and can influence the development of microorganisms. Assessments of receipts show BPA concentrations of up to $30,000 \mathrm{mg} / \mathrm{kg}$ and the new obligation to provide receipts is expected to increase the use of thermal paper. Though only a moderate increase in the use of thermal paper for receipts is anticipated for Austria as a whole, nevertheless a greater number of people will be affected by the permanent contact with receipts, and recycled receipts can contaminate the new paper products with color developers. Although receipts only account for a small percentage of the paper collected for recycling, due to the high concentration of color developers many studies consider thermal paper to be the main cause of exposure to BPA.

\section{Einleitung}

In Österreich gilt seit dem 01.01.2016 die Belegerteilungspflicht. Demnach müssen UnternehmerInnen für jede Barzahlung, darunter fallen auch Zahlungen mit EC- oder Kreditkarten, einen Beleg ausstellen und dem Kunden aushändigen. Der Kunde ist wiederum verpflichtet, den Beleg entgegenzunehmen und bis außerhalb der Geschäftsräum- lichkeiten mit sich $\mathrm{zu}$ führen (BGBl. Nr. 194/1961 i. d. F. 2016).

Aufgrund massiver Proteste verschiedenster Organisationen und Verbände existieren inzwischen Erleichterungen hinsichtlich der Belegerteilungspflicht. Ausgenommen sind grundsätzlich Umsätze im Freien bis zu einem Jahresumsatz von 30.000 Euro sowie Warenausgabe- und Dienstleistungsautomaten, bei denen der Einzelumsatz 20 Euro nicht übersteigt (z. B. Flipper oder Münzprägeautomaten). Des Weiteren entfällt die Belegerteilungspflicht für Fahrausweisautomaten unter der Voraussetzung, dass auf dem Fahrausweis entsprechende Merkmale nach den Anforderungen des $\S 132$ a BAO (Bundesabgabenordnung) erscheinen (BMF 04.08.2016).

Insbesondere kommt bei der Diskussion um die Belegerteilungspflicht und deren Auswirkungen den sogenannten Thermopapieren eine wichtige Rolle zu. Diese Form von Spezialpapieren findet häufig als Kassenbeleg Verwendung (Lassen et al. 2011). Das Druckbild entsteht bei Thermopapieren durch die Übertragung von Hitze beim Druckvorgang (Koehler Paper Group o.J.). Neben den eigentlichen Farbstoffen im Papier beinhalten Thermopapiere einen Farbentwickler, häufig Bisphenol $\mathrm{A}^{1}$ (BPA) (Eckardt und Simat 2015). Diese endokrin wirksame Substanz wird unter anderem in Verbindung mit Herz-Kreislauf-Erkrankungen, Diabetes, Frühgeburten und Fettleibigkeit gebracht (Rochester 2013).

Gelangen Thermopapiere in den Recyclingkreislauf, so ist mit einer Kontamination der hergestellten Recyclingpapiere durch Farbentwickler zu rechnen. Studien wähnen deshalb Thermopapiere als Hauptverursacher für die Verunreinigung von Papierprodukten mit BPA (Gehring et al. 2004; Pivnenko et al. 2015).

1 4,4'-Isopropylidendiphenol, CAS-Nr.: 80-05-7. 
Tab. 1 BPA Belastungen von Thermopapieren

\begin{tabular}{l|l|l|l|l|l|}
$\begin{array}{l}\text { Untersuchungs- } \\
\text { gegenstand }\end{array}$ & $\begin{array}{l}\text { Geografischer } \\
\text { Bezug }\end{array}$ & $\begin{array}{l}\text { BPA } \\
\text { [mg/kg] }\end{array}$ & $\begin{array}{l}\text { BPA }>10.000 ~ \mathbf{~ m g / k g} \\
\text { (Anzahl) }\end{array}$ & $\begin{array}{l}\text { Proben- } \\
\text { anzahl }\end{array}$ & Referenz \\
\hline Thermopapiere im Altpapier & Deutschland & $<1-17.220$ & 5 & 8 & Harling et al. 2012 \\
\hline Unbedruckte Kassenbelege & USA & $<900-17.000$ & 6 & 10 & Mendum et al. 2011 \\
\hline Kassenbelege & Dänemark & $<2-17.600^{*}$ & 6 & 13 & Pivnenko et al. 2015 \\
\hline Kassenbelege & Schweiz & $<0,5-17.000$ & 8 & 10 & Biedermann et al. 2010 \\
Kassenbelege & Belgien & $<0,01-20.900$ & 30 & 44 & Geens et al. 2012
\end{tabular}

* Hinweis, dass Konzentration auf Trockenmasse bezogen wurde. In den restlichen Studien waren die Konzentrationsangaben diesbezüglich undefiniert.

Die vorliegende Arbeit stellt die mit der Verwendung von Kassenbelegen aus Thermopapier verbundenen Gefahren unter Umwelt- und Gesundheitsaspekten in den Fokus. Inwieweit die Anfang des Jahres in Kraft getretene Belegerteilungspflicht mögliche Gefährdungen erhöht, ist ein weiterer Bestandteil dieser Ausarbeitung. Sie soll den derzeitigen Stand der Wissenschaft zur Thematik aufzeigen und verdeutlicht an einem Beispiel aus dem Gastronomiebereich die möglichen Mengen an BPA, welche in den Recyclingkreislauf von Papier gelangen können.

\section{Thermopapiere als Kassenbelege}

Thermopapiere finden in den verschiedensten Bereichen Anwendung. So bestehen Kassenbelege, Tickets, Lotteriescheine, Etiketten und Faxpapiere aus dieser Papiersorte. Gründe für die Verwendung von Thermopapieren sind vor allem in der Zuverlässigkeit der Drucker, den geringen Druckkosten, da keine Druckertinte oder Toner benötigt wird, dem geräuschlosen Druckvorgang und der Kompaktheit der Druckgeräte zu sehen (Lassen et al. 2011).

Eine Studie geht davon aus, dass 80 bis $95 \%$ aller Kassenbelege auf dem dänischen Markt aus Thermopapieren bestehen (Lassen et al. 2011). Der Marktanteil in Österreich sollte in einem ähnlich hohen Bereich liegen (Schmölzer 2016).

Thermopapiere bestehen im Wesentlichen aus einem Basispapier und einer thermosensitiven Schicht (Koehler Paper Group o.J.). Diese enthält unter anderem einen sogenannten Leukofarbstoff, ein unter Raumtemperatur farbloser Farbstoff, sowie einen häufig phenolhaltigen Farbentwickler. Durch Wärmeübertragung reagieren Farbentwickler und Farbstoff miteinander, was zur Farbausbildung und damit zur Entwicklung des Druckbildes führt. Der Farbentwickler fungiert bei dieser Reaktion als Protonendonator (Burkin- shaw et al. 1998; Mendum et al. 2011). Die Wärmebereitstellung erfolgt durch einen mit vielen kleinen Heizelementen besetzten Thermodruckkopf, die eine selektive Erwärmung spezifischer Abschnitte des Thermopapiers ermöglichen (Koehler Paper Group o.J.).

\section{Belastungen durch Farbentwickler}

\subsection{Kassenbelege}

Thermopapiere erfordern, wie bereits erwähnt, die Verwendung eines Farbentwicklers. Eine amerikanische Studie hat insgesamt 19 Substanzen identifiziert, die als Farbentwickler in Thermopapieren bereits zum Einsatz kommen oder deren Einsatz denkbar wäre. Häufig beinhalten Thermopapiere die phenolhaltige Substanz BPA als Farbentwickler (EPA 2015).

Die BPA-Belastungen von Kassenbelegen aus Thermopapieren wurden in mehreren Studien untersucht (Tab. 1).

Die Untersuchungsergebnisse zeigen, dass zum Teil sehr hohe Konzentrationen von bis $\mathrm{zu} 20.900 \mathrm{mg} / \mathrm{kg}$ an BPA in Kassenbelegen vorliegen. Ein Großteil der untersuchten Kassenbelege beinhaltet BPA-Konzentrationen von über $10.000 \mathrm{mg} / \mathrm{kg}$. Es erweist sich hinsichtlich der Höhe der BPA-Konzentration zudem nicht als entscheidend, ob ein Kassenbeleg bedruckt oder unbedruckt ist. Pivnenko et al. (2015) konnten keinen signifikanten Zusammenhang zwischen der bedruckten Fläche eines Kassenbelegs und der BPAKonzentration nachweisen.

Abschn. 3.3 erläutert die Gesundheitsgefahren, welche von Farbentwicklern ausgehen, ausführlich. Dabei wird auch Bezug auf Bisphenol $S^{2}$ (BPS), einen als Alternative zu BPA identifizierten Farbentwickler, genommen. Diese ebenfalls phenolhaltige Substanz

2 4,4'-Sulfonyldiphenol, CAS-Nr.: 80-09-1. besitzt eine chemisch ähnliche Struktur wie BPA (EPA 2015).

Bei einer Untersuchung von Kassenbelegen aus Thermopapier von Apfelbacher et al. (2014) konnte in der einen Hälfte der Proben BPA und in der anderen Hälfte BPS nachgewiesen werden. Diese Feststellung zeigt, dass BPS mittlerweile als alternativer Farbentwickler zu BPA eingesetzt wird. Eine weitere Untersuchung aus Dänemark bestätigt diese Aussage. Kassenbelege mit nur geringen oder keinen BPABelastungen, enthielten hohe Konzentrationen an BPS (Pivnenko et al. 2015). Eine 124 Proben umfassende Studie aus der Schweiz untersuchte Kassenbelege und konnte dabei weitere Substitute für BPA identifizieren. In einem geringeren Anteil der Proben befand sich das bereits erwähnte BPS (3\%), aber auch die Substanzen Pergafast ${ }^{\circledR} 201^{3}(9 \%)$, ein Harnstoff-Derivat, und D-8 ${ }^{4}$ (7\%). Ein Großteil der Proben (81 \%) enthielt weiterhin BPA (Goldinger et al. 2015). Mit dem Ziel, eine Marktübersicht über die derzeit verwendeten Farbentwickler in Thermopapieren $\mathrm{zu}$ erstellen, analysierten Eckardt und Simat (2015) 114 Thermopapier-Proben (Kassenbelege sowie Fahr- und Eintrittskarten) in Sachsen, Deutschland. Rund die Hälfte $(48,2 \%)$ der Thermopapiere enthielt BPA. Der am häufigsten alternativ eingesetzte Farbentwickler war Pergafast ${ }^{\circledR}$ 201 (34,2 \%). In einem geringeren Anteil der Proben befand sich BPS (11,4\%) und D-8 (6,2 \%). Es zeigte sich insgesamt, dass die großen Supermarktketten und Discounter fast ausschließlich BPA-freie Kassenbelege verwenden. Lediglich kleine Geschäfte und Restaurants nutzen noch Kassenbelege, die BPA beinhalten (Eckardt und Simat

${ }^{3} \mathrm{~N}$-(p-Toluolsulfonyl)- $\mathrm{N}^{\prime}$-(3-(p-toluolsulfonyloxy)phenyl)harnstoff, $\quad$ CAS-Nr.: 232938-43-1.

4 4-Hydroxyphenyl-4 0 -isopropoxyphenyl-sulfon, CAS-Nr.: 95235-30-6. 
Tab. 2 Konzentrationen von BPA, BPS, Pergafast ${ }^{\circledR} 201$ und D-8 in Thermopapieren

\begin{tabular}{|l|l|l|l|l|}
\hline Farbentwickler & \multicolumn{2}{l}{$\begin{array}{l}\text { Goldinger et al. (2015) } \\
\text { Konzentrations- } \\
\text { bereich }\end{array}$} & Median & \multicolumn{2}{l}{$\begin{array}{l}\text { Eckardt und Simat (2015) } \\
\text { Konzentrations- } \\
\text { [mg/kg] }\end{array}$} & {$[\mathrm{mg} / \mathrm{kg}]$} & $\begin{array}{l}\text { Median } \\
\text { bereich } \\
{[\mathrm{mg} / \mathrm{kg}]}\end{array}$ & {$[\mathrm{mg} / \mathrm{kg}]$} \\
\hline BPA & $5600-30.400$ & 14.500 & $4000-32.400$ & 15.900 \\
\hline BPS & $8300-12.600$ & 10.000 & $10.500-18.300$ & 13.100 \\
\hline Pergafast ${ }^{\circledR} 201$ & $3300-8200$ & 4600 & $3800-19.100$ & 10.500 \\
\hline D-8 & $3400-13.200$ & 12.000 & $3000-14.700$ & 10.000 \\
\hline
\end{tabular}

2015). Für den österreichischen Markt ergibt sich ein ähnliches Bild. Großverbraucher (Verbrauch von mehr als 50.000 Rollen/Jahr), wie beispielsweise Supermarktketten, setzen zu $40 \% 100 \%$ phenolfreies Thermopapier ein. Weitere $40 \%$ der verwendeten Thermopapiere sind BPA-frei. Die verbleibenden $20 \%$ beinhalten BPA als Farbentwickler. Kleinverbraucher nutzen vor allem BPA-haltige Kassenbelege. BPA-freie (30\%) und $100 \%$ phenolfreie Papiere (10\%) finden nur in geringen Anteilen Anwendung (Schmölzer 2016).

Goldinger et al. (2015) sowie Eckardt und Simat (2015) konnten in ihren Studien ähnlich hohe Konzentrationen für BPA sowie die alternativen Farbentwickler nachweisen (Tab. 2). Lediglich die Maximalkonzentrationen von Pergafast ${ }^{\circledR} 201$ unterscheiden sich um rund $11.000 \mathrm{mg} / \mathrm{kg}$. Die Ergebnisse der beiden Studien weisen aber auch darauf hin, dass Thermopapier-Hersteller BPA bis zu einer zweifach höheren Maximalkonzentration als die restlichen Farbentwickler einsetzen (Goldinger et al. 2015; Eckardt und Simat 2015).

\subsection{Recycling}

Altpapier stellt einen wesentlichen Rohstoff bei der Papierproduktion dar. Das belegt die Einsatzquote von Altpapier in der österreichischen Papierindustrie, die 2014 bei 47,3\% lag (Austropapier 2016). Der Einsatz von Altpapier bei der Papierherstellung ist auch aufgrund von deutlichen Wasser- und Energieeinsparungen im Vergleich zur Herstellung aus Primärfasern vorteilhaft.

Pivnenko et al. (2015) führten Sortieranalysen von separat gesammeltem Altpapier sowie von Papier im Restabfall durch und untersuchten verschiedene Papierfraktionen auf BPA und dessen Strukturanaloge. In der eigens ausgewiesenen Kategorie für Kassenbelege wurden die höchsten Konzentrationen an BPA (bis zu $8300 \mathrm{mg} / \mathrm{kg}$ Trockenmas- se) nachgewiesen. In allen anderen Papierfraktionen war ebenfalls BPA nachweisbar, was auf die Verbreitung von BPA während des Recyclings zurückzuführen ist. Ein Großteil der untersuchten Papierfraktionen enthielt ebenso BPS, wobei die höchsten Konzentrationen (bis zu $210 \mathrm{mg} / \mathrm{kg}$ Trockenmasse) wiederum in der Fraktion der Kassenbelege vorlagen. Die Konzentration aller weiteren untersuchten Strukturanaloge von BPA in Kassenbelegen befand sich unter der Nachweisgrenze (Pivnenko et al. 2015).

Grundsätzlich enthielt die Altpapierfraktion im Restabfall einen höheren Anteil (0,5 \%) an Kassenbelegen als das separat gesammelte Altpapier (0,005\%). Ein größerer Anteil der Kassenbelege befindet sich damit im Restabfall (Pivnenko et al. 2015). Dies bestätigt auch ein Bericht des Joint Research Centre (JRC), wonach nur rund $15 \%$ der Kassenbelege aus Thermopapier in das Altpapier-Recycling gelangen (JRC 2008).

Trotzdem gelten Kassenbelege als Haupteintragsquelle für BPA und BPS in den Papierkreislauf, da die beiden Substanzen fast ausschließlich in Thermopapieren Verwendung finden (Harling et al. 2012; Pivnenko et al. 2015). BPA und BPS weisen eine hohe Affinität für die Festphase auf. Für das Recycling von Papier bedeutet diese Tatsache, dass die erwähnten Farbentwickler mit großer Wahrscheinlichkeit an den Papierfasern anhaften. Zu kurze Papierfasern werden mit dem Schlamm abgeschieden. Längere Fasern verbleiben jedoch im Produktionsablauf und können somit inklusive der anhaftenden Farbentwickler in neue Produkte aus Altpapier gelangen. BPS zeigte gleichzeitig eine hohe Affinität für die gen über das mögliche Verhalten beim Recycling erschwert (Pivnenko et al. 2015). Wasserphase, was verlässliche Aussa-
Studien untersuchten bereits die Eliminationsraten für $\mathrm{BPA}$ während des Papierrecyclings näher. Ein Recyclingprozess mit einer Deinking-Stufe und Thermopapier-Zuschnitten als Inputmaterial erreichte eine Abtrennleistung von $95 \%$ für BPA (JRC 2008). In der Produktion von Kartons konnte während des Deinkings eine Abreicherung von 54 bis $66 \%$ für BPA beobachtet werden. Die Autoren wiesen aber darauf hin, dass es sich hierbei aufgrund von Inhomogenitäten bei der Probenahme und Probenteilung nur um Orientierungswerte handelt. Im unbedruckten Rohkarton, dem Endprodukt, lag im Mittel eine Konzentration von $10 \mathrm{mg} / \mathrm{kg}$ Karton an BPA vor (Harling et al. 2012). Eine weitere Papierfabrik, die Verpackungen aus Wellpappe produziert, verfügte über einen Pulper, der lediglich $10 \%$ des BPA entfernte. Ein Großteil des BPA verbleibt somit in den Recyclingprodukten (JRC 2008). Der Abscheidegrad von BPA während des Recyclingprozesses unterscheidet sich somit je nach eingesetzter Verfahrenstechnik. Toilettenpapier aus $100 \%$ Recyclingpapier enthielt ebenfalls nennenswerte BPA-Konzentrationen von 3,2 bis $46,1 \mathrm{mg} / \mathrm{kg}$ Trockenmasse und stellt damit eine mögliche Quelle für endokrin wirksame Stoffe im Abwasser dar (Gehring et al. 2004).

Weitere Bedenken bestehen hinsichtlich der Abwassereinleitungen von Papier- und Recyclingfabriken in Gewässern. Wasser- und Sedimentproben von Gewässern, die Abwassereinleitungen von Papier- und Recyclingfabriken erhalten, enthielten zum Teil BPAKonzentrationen von bis $\mathrm{zu} 4,1 \mu \mathrm{g} / \mathrm{l}$ im Wasser und 2,2 $\mu \mathrm{g} / \mathrm{g}$ im Sediment (Terasaki et al. 2007). Studien zeigen, dass BPA die sexuelle Entwicklung und Fortpflanzung von Gewässer- und Bodenorganismen beeinflusst (JRC 2008).

\subsection{Gefahren für die Gesundheit}

BPA ist sogenannter endokriner Disruptor, der die Hormonaktivität beeinflusst und Beeinträchtigungen hervorruft (EFSA 2016). BPA kann bereits bei sehr geringen Dosen Effekte auslösen. Bei Ratten, welche einer Dosis von 0,025 mg/kg Körpergewicht/Tag BPA ausgesetzt wurden, entwickelten sich Veränderungen an den Brustdrüsen (Mandrup et al. 2016). In Kassenbelegen befinden sich BPA-Konzentrationen von bis zu $30.400 \mathrm{mg} / \mathrm{kg}$ (Goldinger et al. 2015), die dem 1,2-Millionen- 
Fachen der injizierten Tagesdosis entsprechen. Dieser Vergleich soll die Bedenken hinsichtlich der Verwendung von Thermopapieren unterstreichen.

BPA wird mit einer Reihe von gesundheitlichen Problemen in Verbindung gebracht. Die Literaturstudie von Rochester (2013) weist unter anderem auf Fettleibigkeit, Typ-2-Diabetes, HerzKreislauf-Erkrankungen sowie Fruchtbarkeits- und Leberprobleme hin. Weiterhin steht BPA im Verdacht, das Immunsystem $\mathrm{zu}$ beeinträchtigen oder Frühgeburten $\mathrm{zu}$ verursachen (Rochester 2013).

Die in der chemischen Struktur ähnliche Substanz BPS zeigte in einer Invitro-Studie eine ähnliche hormonelle Wirkung wie BPA (Goldinger et al. 2015). Mit BPS sind damit gleichartige Gesundheitsgefahren verbunden wie mit BPA (Chen et al. 2016). Die Untersuchung von D-8 und Pergafast ${ }^{\circledR} 201$ ließen in der gleichen In-vitro-Studie von Goldinger et al. (2015) keine Beeinflussung des Hormonsystems erkennen. Es wurde jedoch angemerkt, dass weitere Studien notwendig sind, um diese Aussage zu verifizieren (Goldinger et al. 2015).

Besondere Bedeutung findet die Belastung von Thermopapieren mit Bisphenolen im Einzelhandel, da das Kassenpersonal durch den Umgang mit Kassenbelegen ständig dem Schadstoff ausgesetzt ist. Untersuchungen des Urins von Kassierer/innen zeigen deutlich erhöhte BPA-Konzentrationen im Vergleich zu anderweitig tätigen Personen (Thayer et al. 2016). Apfelbacher et al. (2014) verdeutlichen mit ihren Ergebnissen die Relevanz von Thermopapieren im Einzelhandel. Im Körper einer im Einzelhandel tätigen Person in den USA befindet sich $30 \%$ mehr BPA als bei einer durchschnittlichen erwachsenen Person (Apfelbacher et al. 2014).

BPA liegt in Kassenbelegen nicht chemisch gebunden vor, es kann daher leicht bei Berührung auf die Haut übertragen werden (Schreder 2010). Durchschnittlich wird 1,1 $\mu \mathrm{g}$ BPA durch das Halten eines Kassenbons auf einen Fingerballen übertragen. Feuchte oder fettige Haut führte zu einem bis zu 10fach höheren Wert (Biedermann et al. 2010). Eine durchschnittliche Person mit einem Körpergewicht von $70 \mathrm{~kg}$ ist durch $445 \mathrm{ng}$ BPA/Tag aufgrund des Kontakts mit Thermopapieren exponiert. Für das Kassenpersonal liegt dieser Wert vermutlich weitaus höher
(Geens et al. 2012). Ein Worst-Case-Szenario schätzt die Aufnahme von BPA über die Haut auf $250 \mu \mathrm{g} / \mathrm{Tag}$. Diese Berechnung setzt aber unter anderem voraus, dass Kassenbelege mit feuchten Fingern berührt werden (Lassen et al. 2011). Eine andere Studie beziffert die über die Haut aufgenommene Menge an BPA auf 7,1 bis 42,6 $\mu \mathrm{g} /$ Tag (Mielke et al. 2011).

Die BPA-Exposition durch die Nahrung ist mehr als doppelt so hoch wie die durch Thermopapiere (Geens et al. 2012), da sich BPA zum Beispiel in der Innenbeschichtung von Konservendosen befindet (UBA 2010). Denkbar wäre die Migration von BPA aus Recyclingkartons in Richtung von Lebensmitteln, die sich in einer Kartonverpackung befinden. Ein Übergang von BPA aus der Verpackung (Recyclingkarton) auf Lebensmittel konnte bisher in einem Lagerungsversuch von 9 Monaten Dauer ausgeschlossen werden (Harling et al. 2012). Dennoch erwiesen sich Kassenbelege als zusätzliche, nicht zu vernachlässigende Quelle von BPA für den Menschen (Geens et al. 2012).

Thermopapierhersteller wie die Koeh ler Paper Group halten allerdings die Risiken bei der Verwendung von BPA als Farbentwickler in Thermopapieren als unbedenklich. Negative Einflüsse auf die Gesundheit seien nicht nachweisbar, da entsprechende Studienergebnisse bisher nicht reproduziert werden konnten, so die Aussage (Koehler Paper Group 2016). Dabei stützt sich Koehler auch auf Aussagen des Bundesinstituts für Risikobewertung (BfR), welches sich wiederum auf Bewertungen der Europäischen Behörde für Lebensmittelsicherheit (EFSA) beruft. BPA stellt demnach keine Gefährdung für die menschliche Gesundheit dar, da die über verschiedene Quellen (z. B. Thermopapier oder Konservendosen) aufgenommenen Mengen als unbedenklich gelten. Aufgrund neuer Erkenntnisse wurde jedoch die tolerierbare tägliche Aufnahmemenge (TDI) von $50 \mu \mathrm{g} / \mathrm{kg}$ Körpergewicht/Tag auf $4 \mu \mathrm{g} / \mathrm{kg}$ Körpergewicht/Tag herabgesetzt. Die Senkung des TDI begründet die EFSA damit, dass Niedrigdosiseffekte bisher nicht verlässlich nachgewiesen wurden und diesbezüglich Unsicherheiten bestehen (BfR 2015).

In Österreich wurde mittlerweile der Bundesregierung ein Entschließungsantrag vorgelegt, der ein Verbot von BPA und BPS in Thermopapieren fordert (Parlament der Republik Österreich
2016). Eine Beschränkung soll auch auf EU-Ebene folgen, wonach Thermopapiere maximal 0,02 Gewichtsprozent BPA enthalten dürfen (ECHA 2014).

\section{Auswirkungen der Belegerteilungspflicht am Beispiel eines österreichischen Gastronomiedienstleisters}

Eine Abschätzung soll die konkreten Auswirkungen der Belegerteilungspflicht erläutern, die sich aus einen möglichen Mehrverbrauch von Kassenbelegen ergeben. Die hierbei verwendeten Verbrauchsdaten stammen von der Österreichischen Mensen Betriebsgesellschaft $\mathrm{mbH}$, die in 60 Betrieben rund 4 Mio. Gäste im Jahr bedient (Wild 2016). Kassenbelege wurden von dem Kassenpersonal bisher nur auf Nachfrage ausgestellt. Die Belegerteilungspflicht erfordert seit 01.01.2016 vom Unternehmen, jedem Gast einen Kassenbeleg auszuhändigen, wobei nur ein Bruchteil der Gäste den Beleg an sich nimmt. Ein Großteil der Gäste lässt den Kassenbeleg in eigens dafür aufgestellten Behältnissen im Kassenbereich zurück, was zur direkten Entsorgung führt, allerdings dem Gesetz widerspricht, dass der Beleg bis außerhalb der Geschäftsräumlichkeiten mit sich zu führen ist. Würde der Beleg von allen Gästen bis außerhalb der Geschäftsräumlichkeiten mitgeführt werden, stellt sich die Frage nach der weiteren Entsorgungsoption außerhalb: Landen dann alle Belege in Abfallbehältern oder werden sie doch eher einfach weggeworfen?

Mit einem durchschnittlichen Gewicht von ca. 0,48 g pro Beleg ergibt sich allein für die Mensen in Österreich ein jährliches Aufkommen an Kassenbelegen von rund $1900 \mathrm{~kg}$. Dieser Wert entspricht dem rund 27-Fachen des Ausgangswertes von vormals rund $71 \mathrm{~kg}$ an Thermopapieren. Damit lässt sich der Mehrverbrauch durch die Belegerteilungspflicht auf ca. $1829 \mathrm{~kg}$ Thermopapier beziffern. Bei dem verwendeten Thermopapier handelt es sich laut Zulieferer um ein Standardpapier, welches BPA als Farbentwickler enthalten kann. Die Mehrkosten von Thermopapieren ohne BPA liegen bei ca. $10 \%$ (Schmölzer 2016) bzw. mehr als dem Doppelten (Wild 2016). Die Bäckereikette „Baguette“ rechnet mit Mehrkosten im Bereich von 40.000 bis 50.000 Euro für die Anschaffung von Kassenrollen für das laufende Jahr. Wei- 


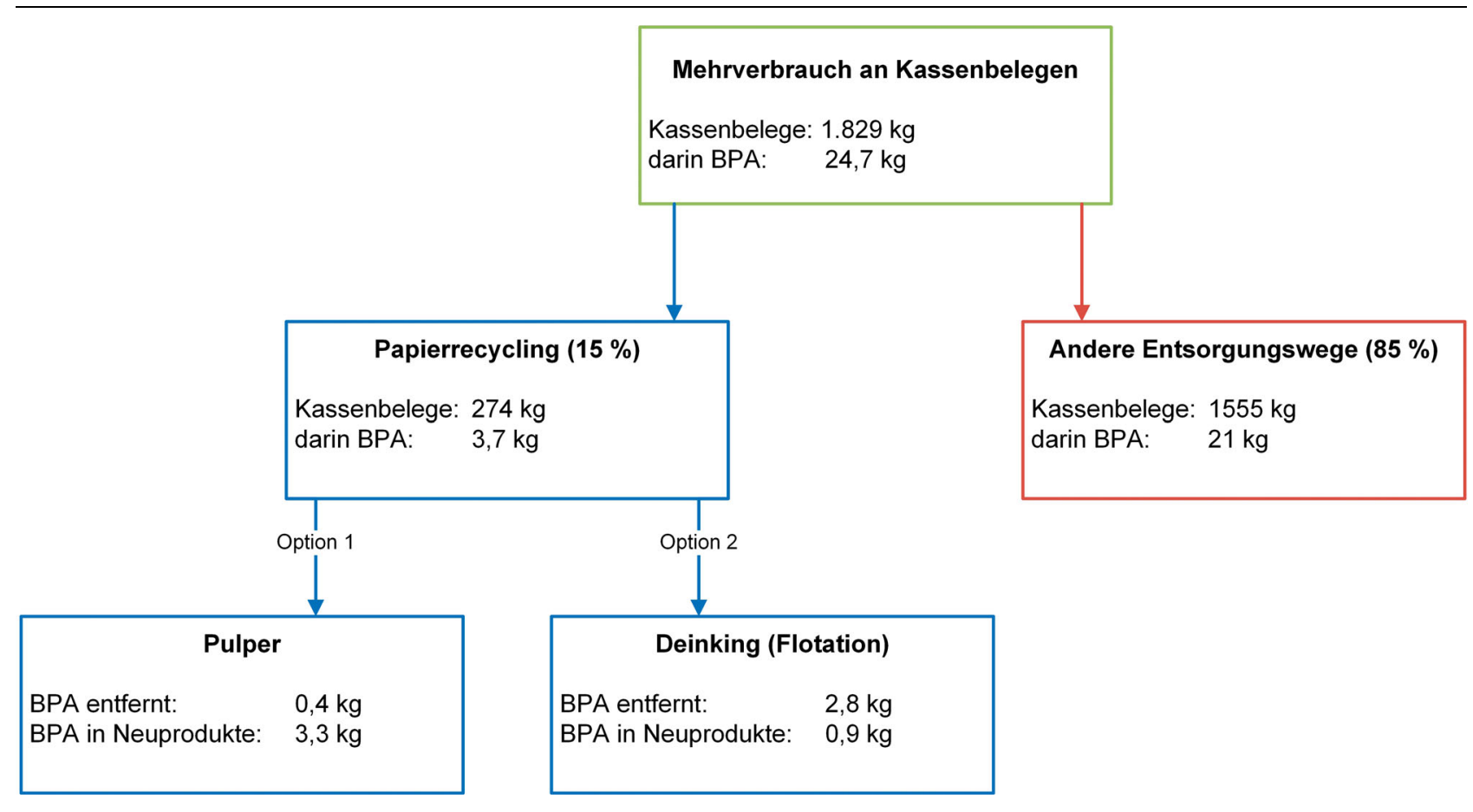

Abb. 1 Aufkommen an Kassenbelegen, darin enthaltenes BPA und Entsorgungswege

terhin zeigt sich in deren Filialen ein vermehrter Reinigungsaufwand für die Beseitigung der von den Kunden auf den Boden geworfenen Kassenbelege (Mair 2016).

Auf Grundlage der Angaben wurden für die Mensen in Österreich die Mengen an BPA (Abb. 1) ermittelt, welche aufgrund des Mehrverbrauchs an Kassenbelegen in den Recyclingkreislauf und damit in die Produktion neuer Papierprodukte gelangen. Die Studie von Goldinger et al. (2015) diente aufgrund ihrer großen Anzahl an untersuchten Proben als Referenz für die BPA-Konzentrationen in Kassenbelegen. Für die zusätzlich verbrauchten Kassenbelege ergibt sich bei einer mittleren BPA-Konzentration von $13.500 \mathrm{mg} / \mathrm{kg}$ eine Gesamtmenge von 24,7 kg BPA. Bei einer Maximalkonzentration von $30.400 \mathrm{mg} / \mathrm{kg}$ BPA könnte sogar eine Menge von rund 55,6 kg BPA im errechneten Mehrverbrauch an Kassenbelegen vorliegen.

Nach Literaturangaben erreichen aber nur 15 \% (JRC 2008) der verwendeten Kassenbelege den Recyclingkreislauf, was im Rahmen dieser Abschätzung $274 \mathrm{~kg}$ an Kassenbelegen bzw. $3,7 \mathrm{~kg}$ (Mittelwert) an darin enthaltenem BPA entspricht. Während des Recyclingprozesses erfolgt je nach integrierter Verfahrenstechnik, wie unter Abschn. 3.2 beschrieben, eine weite- re Abreicherung von BPA im Bereich von 10 bis $95 \%$ (Harling et al. 2012; JRC 2008). Die Berechnungen berücksichtigen einen BPA-Abscheidegrad von $10 \%$ für einen Pulper sowie einen Abscheidegrad von $75 \%$ für das Deinking (errechneter Mittelwert aus den Literaturangaben von Harling et al. (2012) und JRC (2008)). Es gelangen somit $3,3 \mathrm{~kg}$ BPA (Pulper) bzw. 0,9 kg BPA (Deinking) in Produkte aus Recyclingpapier (Abb. 1).

\section{Schlussfolgerungen und Ausblick}

Die vorliegende Arbeit zeigt, dass die Belegerteilungspflicht im Einzelfall den Verbrauch an Kassenbelegen erheblich steigert und aufgrund der verwendeten Thermopapiere einen weiteren Schadstoffeintrag in den Papierkreislauf bewirken kann. Ein weiterer negativer Aspekt liegt im Ressourcenverbrauch für die Herstellung der im Beispiel der Mensen in Österreich genannten rund $1800 \mathrm{~kg}$ an Thermopapier. Ebenso ergeben sich negative Auswirkungen, wenn die Belege nicht richtig entsorgt, sondern entweder an Ort und Stelle in den Geschäftsräumlichkeiten oder davor weggeworfen werden und ein zusätzlicher Reinigungsaufwand entsteht.

Für ganz Österreich ist jedoch nur von einer moderaten Steigerung des Verbrauchs an Thermopapieren für
Kassenbelege auszugehen. Begründet werden kann diese Tatsache damit, dass beispielweise große Supermarktketten schon immer Kassenbelege ausstellen. Der Mehrverbrauch entsteht eher bei kleinen Läden, wie z. B. Trafiken. Ein in Österreich ansässiger Großhändler schätzt den Mehrverbrauch durch die Belegerteilungspflicht auf 2 bis $5 \%$ ein. Er sieht einen größeren Mehrverbrauch von Thermopapieren eher im Aufdruck von Werbebotschaften auf den Kassenbelegen (Schmölzer 2016). Hier müsste auch hinsichtlich des generellen Ressourcenverbrauchs die Frage nach der Notwendigkeit dieser Werbung gestellt werden.

Die Verwendung von Thermopapieren stellt aber ein generelles Problem dar. Auch wenn die großen Supermarktketten eher auf BPA-freie Thermopapiere zurückgreifen, so zeigen Untersuchungen, dass BPA immer noch der am häufigsten eingesetzte Farbentwickler ist (Eckardt und Simat 2015). Dessen endokrine Wirksamkeit und die damit verbundenen Gesundheitsgefahren sowie Auswirkungen auf das Papierrecycling wurden in dieser Arbeit diskutiert. Die alternativen Farbentwickler, vor allem die in der chemischen Struktur BPA-ähnlichen Substanzen, zeigen in Studien ähnliche Wirkungen wie BPA (Chen et al. 2016). D-8 und Pergafast ${ }^{\circledR} 201$ sind noch nicht hin- 
reichend untersucht (Goldinger et al. 2015). Unter Umwelt- und Gesundheitsaspekten existieren derzeit somit keine Alternativen zu BPA (EPA 2015). Thermopapierhersteller, EFSA und BfR sehen allerdings im Gegensatz dazu keine Risiken für die Gesundheit, die sich durch die Verwendung von BPA in Thermopapieren ergeben. Die über verschiedene Quellen aufgenommenen Mengen an BPA seien unbedenklich. Durch die deutliche Herabsetzung des TDI von $50 \mu \mathrm{g} / \mathrm{kg}$ Körpergewicht $/ \mathrm{Tag}$ auf $4 \mu \mathrm{g} / \mathrm{kg}$ Körpergewicht/Tag zeigt sich jedoch, dass die Bedenken der Behörden bezüglich BPA größer werden (Koehler Paper Group 2016; BfR 2015)

Eine vielversprechende Entwicklung stellt möglicherweise das Thermopapier „Ökobon“ dar. Dieses beinhaltet bereits eine schwarze Farbschicht, welche von einer thermosensitiven Schicht mit feinen Bläschen abgedeckt ist. Durch die Hitzeübertragung mittels Thermodruckkopf kollabieren die Bläschen und die darunterliegende schwarze Farbschicht tritt zum Vorschein. Die Verwendung eines Farbentwicklers erfordert dieses Thermopapier nicht (Ökobon GmbH 2016).

Nach Expertenmeinungen verhindern allerdings Qualitäts- und Preisgründe den Durchbruch dieses Thermopapiers. Die im Papier eingearbeiteten feinen Bläschen können bereits bei der Produktion, bei der das Papier über Transportwalzen und -rollen geführt wird, platzen. Dieser Vorgang führt zu einer leichten Graufärbung des Papiers, was wiederum Kontrastprobleme bei der Schrift und Probleme beim Druck bedingt, da Druckersensoren das grau gefärbte Papier nicht erkennen (Schmölzer 2016).

Digitale Kassenbelege erweisen sich als weitere Alternative für Quittungen in Papierform. Bei einem Teil solcher Systeme erfolgt die Zusendung des Kassenbelegs an eine vorher bekanntgegebene E-Mail-Adresse. Ein anderer Teil verwendet eine entsprechende Smartphone-App, die einen persönlichen Code erzeugt. Das Kassenpersonal scannt diesen Code und der Kassenbeleg gelangt anschließend auf das Smartphone. Allerdings stehen Systeme für digitale Kassenbelege in der Kritik, da sie das Sammeln kundenspezifischer Daten ermöglichen. Unternehmen könnten die erhobenen Daten wiederum nutzen, um beispielsweise das Kaufverhalten der Kunden zu analysieren (Müller 2014). Möglich wäre daher auch ein elektronisches Kassensystem, bei dem die Belegerstellung nur auf Nachfrage erfolgt und ansonsten komplett auf einen Ausdruck sowie elektronische Weiterleitung der Daten verzichtet wird.
Generell wären Informationskampagnen zur empfohlenen Entsorgung von Thermopapier über den Restabfall sinnvoll, um die Qualität beim Papierrecycling hoch zu halten und keine sonstige Verbreitung der enthaltenen Schadstoffe zu ermöglichen.

Abschließend lässt sich aufgrund der hier erfolgten Diskussion feststellen, dass die Belegerteilungspflicht in der jetzigen Form zu verstärkten Auswirkungen auf die Umwelt und Gesundheit geführt hat.

Open access funding provided by University of Innsbruck and Medical University of Innsbruck.

\section{Interessenkonflikt}

M. Genslein und A. Bockreis geben an, dass kein Interessenkonflikt besteht.

Open Access Dieser Artikel wird unter der Creative Commons Namensnennung 4.0 International Lizenz (http:// creativecommons.org/licenses/by/4.

$0 /$ deed.de) veröffentlicht, welche die Nutzung, Vervielfältigung, Bearbeitung, Verbreitung und Wiedergabe in jeglichem Medium und Format erlaubt, sofern Sie den/die ursprünglichen $\mathrm{Au}$ tor(en) und die Quelle ordnungsgemäß nennen, einen Link zur Creative Commons Lizenz beifügen und angeben, ob Änderungen vorgenommen wurden.
Apfelbacher, M., Cioci, M., Strong, P. (2014): BPA and BPS in thermal paper: Results of testing in Minnesota hospitality industry. Hg. v. Minnesota Pollution Control Agency. Saint Paul. Online verfügbar unter https://www.pca.state.mn.us/ sites/default/files/p-p2s10-13.pdf, zuletzt geprüft am 05.05.2016.

Austropapier (2016): Papier aus Österreich Branchenbericht 2015/16. Online verfügbar unter http://www.austropapier.at/fileadmin/ austropapier.at/dateiliste/Dokumente/

Downloads/Jahresberichte/00_Gesamt al

klein.pdf, zuletzt geprüft am 21.07.2016.

BGBI. Nr. 194/1961: Bundesgesetz über allgemeine Bestimmungen und das Verfahren für die von Abgabenbehörden des Bundes, der Länder und Gemeinden verwalteten Abgaben (Bundesabgabenordnung - BAO), in der Fassung vom 04.07.2016.

Biedermann, S., Tschudin, P., Grob, K. (2010): Transfer of bisphenol A from thermal printer paper to the skin. In: Anal Bioanal Chem 398 (1), S. 571-576. DOI: 10.1007/s00216-010-3936-9. BMF (Bundesministerium für Finanzen) (04.08.2016): Erlass zur EinzelaufzeichnungsRegistrierkassen- und Belegerteilungspflicht, Erlass des BMF vom 04.08.2016. Fundstelle: Findok-Nr. 72122.1. Online verfügbar unter https:// findok.bmf.gv.at $/$ findok? execution $=$ e100000s $1 \&$ dokumentId=4b9801d0- c0c1- 4 d0a-b3ef60a20743732f, zuletzt geprüft am 11.08.2016 BfR (Bundesinstitut für Risikobewertung) (2015): Fragen und Antworten zu Bisphenol A in verbrauchernahen Produkten. Online verfügbar unter http://www.bfr.bund.de/de/fragen_und antworten_zu_bisphenol_a_in_verbraucher nahen_produkten-7195.html, zuletzt geprüft am 16.08.2016.

Burkinshaw, S. M., Griffiths, J., Towns, A. D. (1998): Reversibly thermochromic systems based on pH-sensitive functional dyes. In: J. Mater. Chem. 8 (12), S. 2677-2683. DOI: $10.1039 / \mathrm{a} 805994 \mathrm{~b}$.

Chen, D., Kannan, K., Tan, H., Zheng, Z., Feng, Y.-L., Wu, Y., Widelka, M. (2016): Bisphenol analogues other than BPA: Environmental occurrence, human exposure, and toxicity-A review. In: Environmental science \& technology 50 (11), S. 5438-5453. DOI: 10.1021/acs.est.5b05387. ECHA (2014): Annex XV Restriction Report. Proposal for a restriction. Substance name: 4.4'isopropylidenediphenol (bisphenol A; BPA). Online verfügbar unter http://echa.europa.eu/ documents/10162/c6a8003c-81f3-4df6-b7e815a3a36baf76, zuletzt geprüft am 18.07.2016.
Eckardt, M., Simat, T. (2015): Phenolische und nicht-phenolische Farbentwickler sowie extrahierbare Sensitizer in Thermopapieren - eine Marktübersicht. Technische Universiät Dresden (Poster). Online verfügbar unter https://www. chm.tu-dresden.de/lc2/dateien/2015_Eckardt_ Poster_Thermopapier.pdf, zuletzt geprüft am 02.08.2016.

EPA (United States Environmental Protection Agency) (2015): Bisphenol A alternatives in thermal paper. Online verfügbar unter https:// www.epa.gov/sites/production/files/2015-08/ documents/bpa_final.pdf, zuletzt geprüft am 22.04.2016.

EFSA (European Food Safety Authority) (2016): Endokrin aktive Substanzen. Online verfügbar unter https://www.efsa.europa.eu/de/topics/ topic/eas, zuletzt geprüft am 02.08.2016.

Geens, T., Goeyens, L., Kannan, K., Neels, H., Covaci, A. (2012): Levels of bisphenol-A in thermal paper receipts from Belgium and estimation of human exposure. In: Science of The Total Environment 435-436, S. 30-33. DOI: 10.1016/j.scitotenv.2012.07.001.

Gehring, M., Vogel, D., Tennhardt, L., Weltin, D., Bilitewski, B. (2004): Bisphenol A Contamination of Wastepaper, Cellulose And Recycled Paper 
Products. In: WIT Transactions on Ecology and the Environment 78. DOI: 10.2495/WM040291. Ökobon GmbH (2016): Das innovative Funktionsprinzip. Online verfügbar unter http:// ökobon.de/infos.html, zuletzt geprüft am 02.08.2016.

Goldinger, D. M., Demierre, A.-L., Zoller, O. Rupp, H., Reinhard, H., Magnin, R. et al (2015): Endocrine activity of alternatives to BPA found in thermal paper in Switzerland. In: Regulatory Toxicology and Pharmacology 71 (3), S. 453-462. DOI: 10.1016/j.yrtph.2015.01.002

Harling, A., Helling, R., Simat, T., Grob, K. (2012): Ausmaß der Migration unerwünschter Stoffe aus Verpackungsmaterialien aus Altpapier in Lebensmittel. Hg. v. Bundesministerium für Ernährung, Landwirtschaft und Verbraucherschutz. Online verfügbar unter https://service.ble.de/ptdb/index2.php?detail id $=21002 \&$ site $k e y=141 \&$ stichw $=2809 \mathrm{HS} 012 \&$ zeilenzahl_zaehler $=1 \&$ pId=21002\&dId=116619, zuletzt geprüft am 19.02.2016.

JRC (Joint Research Centre) (2008): European Union risk assessment report 4,4'-Isopropylidenediphenol (Bisphenol A). Online verfügbar unter http://publications.jrc.ec.europa.eu/ repository/bitstream/JRC59980/lbna24588enn. pdf, zuletzt geprüft am 25.04.2016.

Lassen, C., Hagen Mikkelsen, S., Brandt, U. K. (2011): Migration of bisphenol A from cash register receipts and baby dummies. Hg. v. Danish Ministry of the Environment. Online verfügbar unter http://www2.mst.dk/udgiv/publications/ 2011/04/978-87-92708-93-9.pdf, zuletzt geprüft am 08.06.2016.

Mair, T. (2016): Registrierkassenpflicht: Bloß nicht verzetteln. In: Tiroler Tageszeitung Magazin, 31.01.2016 (31), S. 18-21. Online verfügbar unter http://www.tt.com/lebensart/11072484 91/registrierkassenpflicht-blo\%C3\%9F-nichtverzetteln.csp, zuletzt geprüft am 04.07.2016.
Mandrup, K., Boberg, J., Isling, L. K., Christiansen, S., Hass, U. (2016): Low-dose effects of bisphenol A on mammary gland developmen in rats. In: Andrology4, S. 673-683. DOI:10.1111/ andr.12193

Mendum, T., Stoler, E., VanBenschoten, H. Warner, J. C. (2011): Concentration of bisphenol A in thermal paper. In: Green Che mistry Letters and Reviews 4 (1), S. 81-86. DOI 10.1080/17518253.2010.502908.

Mielke, H., Partosch, F., Gundert-Remy, U. (2011): The contribution of dermal exposure to the internal exposure of bisphenol A in man. In: Toxicology letters 204 (2-3), S. 190-198. DOI 10.1016/j.toxlet.2011.04.032.

Müller, B. (2014): Kassenbon aufs Handy. In: Süddeutsche Zeitung 2014, 19.09.2014. Online verfügbar unter http://www.sueddeutsche.de/ wirtschaft/elektronische-kassenzettel-kassen

bon-aufs-handy-1.2135096, zuletzt geprüft am 02.08.2016

Koehler Paper Group (o. J.): Produktinformation. Online verfügbar unter https://www. koehlerpaper.com/media/docs/de/produktin formationen/Thermo/Thermo-Produktbro schuere_D.pdf, zuletzt geprüft am 14.17.2016. Koehler Paper Group (2016): FAQs. Online verfügbar unter https://www.koehlerpaper com/de/service/faq.php, zuletzt geprüft am 16.08.2016

Parlament der Republik Österreich (2016): Selbstständiger Entschließungsantrag der Abgeordneten Dr. Dagmar Belakowitsch-Jenewein, Kolleginnen und Kollegen betreffend Verbot von Bisphenol in Thermopapieren. Nationalrat, XXV. Gesetzgebungsperiode (Entschließungsantrag 1806/A(E)). Online verfügbar unter https:// www.parlament.gv.at/PAKT/VHG/XXV/A/A 01806/fname 546160.pdf, zuletzt geprüft am 02.08.2016.
Pivnenko, K., Pedersen, G. A., Eriksson, E., Astrup, T. F. (2015): Bisphenol A and its structura analogues in household waste paper. In: Waste management (New York, N.Y.) 44, S. 39-47. DOI: 10.1016/j.wasman.2015.07.017.

Rochester, J. R. (2013): Bisphenol A and human health: A review of the literature. In: $R e$ productive Toxicology 42, S. 132-155. DOI: 10.1016/j.reprotox.2013.08.008

Schmölzer, P. (2016): Persönliche Mitteilungen Geschäftsführer Schmölzer - mediaJETCAD. Pusarnitz.

Schreder, E. (2010): On the money. BPA on dollar bills and receipts. Online verfügbar unter http://saferchemicals.org/sc/wp-content/ uploads/sites/3/2014/05/OnTheMoneyReport_ Final2.pdf, zuletzt geprüft am 02.08.2016. Terasaki, M., Shiraishi, F., Fukazawa, H., Makino, M. (2007): Occurrence and estrogenicity of phenolics in paper-recycling process water: Pollutants originating from thermal paper in waste paper. In: Environmental Toxicology and Chemistry 26. DOI: 10.1897/06-642R.1.

Thayer, K. A., Taylor, K. W., Garantziotis, S. Schurman, S. H., Kissling, G. E., Hunt, D. et al (2016): Bisphenol A, Bisphenol S, and 4-Hydroxyphenyl 4-Isoprooxyphenylsulfone (BPSIP) in urine and blood of cashiers. In: Environmental health perspectives 124 (4), S. 437-444. DOI: 10.1289/ehp.1409427.

UBA (Umweltbundesamt) (2010): Bisphenol A Massenchemikalie mit unerwünschten Nebenwirkungen. Dessau-Roßlau. Online verfügbar unter https://www.umweltbundesamt.de/sites/ default/files/medien/publikation/long/3782. pdf, zuletzt geprüft am 19.02.2016. Wild, N. (2016): Persönliche Mitteilungen. Prokurist der Österreichischen Mensen Betriebsgesellschaft mbH. Innsbruck. 